\title{
APPROXIMATION OF A NONLINEAR ELLIPTIC PROBLEM ARISING IN A NON-NEWTONIAN FLUID FLOW MODEL IN GLACIOLOGY
}

\author{
Roland Glowinski ${ }^{1}$ And Jacques Rappaz ${ }^{2}$
}

\begin{abstract}
The main goal of this article is to establish a priori and a posteriori error estimates for the numerical approximation of some non linear elliptic problems arising in glaciology. The stationary motion of a glacier is given by a non-Newtonian fluid flow model which becomes, in a first twodimensional approximation, the so-called infinite parallel sided slab model. The approximation of this model is made by a finite element method with piecewise polynomial functions of degree 1 . Numerical results show that the theoretical results we have obtained are almost optimal.
\end{abstract}

Mathematics Subject Classification. 65N15, 76A05.

Received: June 19, 2002. Revised: November 18, 2002.

\section{INTRODUCTION}

In this paper, we consider the stationary motion of an idealized glacier made with an infinite ice mass between two parallel planes. We suppose that the inclination of the glacier is slight and the ice is assumed as being an incompressible viscous fluid. In order to conduct theoretical studies, glaciologists consider often this simplified model, so-called infinite parallel sided slab, and described in Blatter [3] for instance. Let us remark that glacier ice is treated as a non-Newtonian fluid with a nonlinear relationship between the rate deformation tensor and the deviatoric stress tensor like in the Bingham model (see [7] and its references). In Blatter's model, the viscosity is an implicit function of the deformation tensor. The mathematical analysis of this problem is done in Colinge-Rappaz [5] and we will adopt in the following, the formulation of this last paper.

If $x_{1}, x_{2}$ are the two Cartesian coordinates in the plane of the glacier occupying the Lipschitzian domain $\Omega$, we will denote by $u\left(x_{1}, x_{2}\right)$ the horizontal velocity component of the ice at the point $\left(x_{1}, x_{2}\right) \in \Omega$. After a rescaling of the physical velocity of the ice, $u$ will be satisfying the following equation:

$$
-\operatorname{div}(f(|\nabla u|) \nabla u)=p \quad \text { in } \Omega
$$

where $p$ is a hydrostatic pressure force acting on the glacier and $f$ is a function resulting from a constitutive law for the ice. Clearly speaking we have to add some boundary conditions that we take as homogeneous Dirichlet

\footnotetext{
Keywords and phrases. Finite element method, a priori error estimates, a posteriori error estimates, non-Newtonian fluids, infinite parallel sided slab model in glaciology.

1 Department of Mathematics, University of Houston, Houston, TX 77204-3476, USA.

2 Department of Mathematics, EPFL, 1015 Lausanne, Switzerland. e-mail: jacques.rappaz@epfl.ch
} 
conditions for the sake of simplicity:

$$
u=0 \quad \text { on the boundary } \partial \Omega \text {. }
$$

In Blatter's model (see [3] for instance), the function $f:(0,+\infty) \rightarrow(0,+\infty)$ is a smooth function implicitly given by the following relationship:

$$
f(s)^{-1}=(s f(s))^{\frac{\alpha}{1-\alpha}}+T_{0}^{\frac{\alpha}{1-\alpha}}, \quad \forall s \in(0,+\infty),
$$

where $\alpha$ is a parameter belonging to the open interval $(0,1)$ and $T_{0}$ is a positive number. This ice behavior law is used in several computer codes (see [9] for instance). It is a little different from the one given in ColingeRappaz [5] but doesn't change the conclusions obtained in this last paper. We will begin in Section 2 by proving that $f$ defined in (3) satisfies the following properties:

(H1) $f \in C^{1}(0,+\infty) \quad$ and $\quad f^{\prime}(s) \stackrel{\text { def }}{=} \frac{\mathrm{d} f}{\mathrm{~d} s}(s)<0 \quad$ for all $s \in(0,+\infty)$;

(H2) $\exists \beta, \gamma>0$ such that $\beta(1+s)^{-\alpha} \leq f(s) \leq \gamma(1+s)^{-\alpha}, \quad \forall s \in(0,+\infty)$;

(H3) the function $g(s) \stackrel{\text { def }}{=} s f(s)$ is such that there exist $\omega, \rho>0$ satisfying

$$
\omega(1+s+r)^{-\alpha}(s-r) \leq g(s)-g(r) \leq \rho(1+s+r)^{-\alpha}(s-r), \quad \forall s \geq r \geq 0 .
$$

Let us notice that property (H3) is exactly the assumption (A) of Barrett and Liu [2] in which the number $p-2$ is set to $-\alpha$ and the value $\alpha$ taken by Barrett and Liu in [2] is vanishing. A direct consequence is that we will be able to obtain a priori error estimates directly from [2].

It is proven in Colinge-Rappaz [5] that with the properties (H1)-(H3) and if $p \in W^{-1, \frac{2-\alpha}{1-\alpha}}(\Omega)$, where $\Omega$ is a Lipschizian domain, problem $(1,2)$ has a unique solution $u$ in the usual Sobolev space $W_{0}^{1,2-\alpha}(\Omega)$. Actually this solution minimizes the functional

$$
v \rightarrow J(v)=\int_{\Omega}[F(|\nabla v|)-p \cdot v] \mathrm{d} x
$$

where $F(s)$ is a primitive of $s f(s)$ given by $F(s)=\int_{0}^{s} g(\tau) \mathrm{d} \tau$. Observe that property (H3) implies that $F^{\prime \prime}(s) \geq \omega(1+2 s)^{-\alpha}$ for all $s$ in $(0, \infty)$ so that the functional $J$ is strictly convex. Due to this property, it is also shown that if $X_{h}$ is a finite dimensional subspace of $W_{0}^{1,2-\alpha}(\Omega)$ satisfying

$$
\lim _{h \rightarrow 0} \inf _{v_{h} \in X_{h}}\left\|v-v_{h}\right\|_{W_{0}^{1,2-\alpha}(\Omega)}=0 \quad \text { for all } v \in W_{0}^{1,2-\alpha}(\Omega),
$$

then there exists a unique $u_{h} \in X_{h}$ which minimizes $J$ on $X_{h}$ and $\left\|u-u_{h}\right\|_{W_{0}^{1,2-\alpha}(\Omega)}$ converges to zero when $h$ tends to zero. It is the case when we choose for $X_{h}$ the classical finite element subspace of piecewise polynomial functions of degree 1 on a regular triangulation $\Upsilon_{h}$ of $\bar{\Omega}$, vanishing on $\partial \Omega$.

The main goal of this paper is to establish some results concerning a priori and a posteriori error estimates for $\left\|u-u_{h}\right\|$ in the $W_{0}^{1,2-\alpha}(\Omega)$ - norm or in other norms.

For the approximations and error estimates of degenerate or non-degenerate quasilinear elliptic problems, we refer to the Barrett-Liu's paper [2] and all its references. Let us still mention that the error estimates given in [2] improve the ones for the $p$-Laplacian established originally by Glowinski-Marrocco [6]. We also establish a posteriori error estimates in a same way as in Baranger-El Amri [1].

Finally remark that Liu-Yan [8] have recently improved, in some particular cases, a posteriori error estimates for $p$-Laplacian. To do this, they extend a quasi-norm technique which is not trivial and not applied in this paper for obtaining a posteriori error estimates. However we numerically compare our estimator with the Liu-Yan's one. 
An outline of this paper is as follows. In Section 2 we show that the function $f$, implicitly given by (3), satisfies properties (H1), (H2) and (H3) and consequently error estimates for $\left\|u-u_{h}\right\|$ in different norms are obtained from [2]. Sections 3 and 4 are devoted to a priori and a posteriori error estimates respectively. In Section 5 we give some numerical results that confirm these results.

\section{About the Function $f$}

In this paragraph we show that the function $f$ is well defined by (3) and that properties (H1), (H2) and (H3) are fulfilled when $\alpha \in(0,1)$ in the definition of $f$.

Lemma 1. Function $f$ is well defined by (3) and (H1), (H2) hold.

Proof. Let $s$ be fixed in the open interval $(0, \infty)$ and consider the two following functions:

$$
T(y) \stackrel{\text { def }}{=}(s y)^{\frac{\alpha}{1-\alpha}}+T_{0}^{\frac{\alpha}{1-\alpha}}, \quad R(y) \stackrel{\text { def }}{=} 1 / y \quad \text { where } y \in(0,+\infty) .
$$

Clearly speaking, since $\alpha \in(0,1)$, then $T$ is an increasing continuous function on $(0,+\infty)$ and its graph possesses exactly one intersection point with the graph of $R$. We call $y \stackrel{\text { def }}{=} f(s)$ the abscissa of this intersection point and in this way, $f(s)$ satisfies relationship (3). We have $f(s)>0$ when $s \in(0,+\infty)$.

By differentiating (3) with respect to $s$, we obtain:

$$
f^{\prime}(s)\left[\frac{\alpha}{1-\alpha} s(s f(s))^{\frac{2 \alpha-1}{1-\alpha}}+\frac{1}{f(s)^{2}}\right]=-\frac{\alpha}{1-\alpha} f(s)(s f(s))^{\frac{2 \alpha-1}{1-\alpha}}
$$

and consequently $f^{\prime}(s)<0, \forall s \in(0,+\infty)$ which proves that (H1) holds.

Equality (3) shows immediately that $\frac{1}{f(s)} \geq(s f(s))^{\frac{\alpha}{1-\alpha}}$ and $\frac{1}{f(s)} \geq T_{0}^{\frac{\alpha}{1-\alpha}}$. It follows that $f(s) \leq s^{-\alpha}$ and $f(s) \leq T_{0}^{-\frac{\alpha}{1-\alpha}}$ and consequently the upper bound of (H2) is proven. By using this inequality in (3) we obtain:

$$
\frac{1}{f(s)} \leq T_{0}^{\frac{\alpha}{1-\alpha}}+s^{\alpha}
$$

which implies the lower bound of (H2).

Lemma 2. If $g(s)=s f(s)$, then $g$ satisfies (H3).

Proof. We have from (3):

$$
\frac{s}{g(s)}=g(s)^{\frac{\alpha}{1-\alpha}}+T_{0}^{\frac{\alpha}{1-\alpha}} .
$$

By differentiating (8) with respect to $s$ we obtain:

$$
g^{\prime}(s)=\left[\frac{\alpha}{1-\alpha} g(s)^{\frac{\alpha}{1-\alpha}}+\frac{s}{g(s)}\right]^{-1} .
$$

Since $g^{\prime}(s) \geq 0$, then $g(s)^{\frac{\alpha}{1-\alpha}}$ is an increasing function. Moreover $s g(s)^{-1}=f(s)^{-1}$ is also an increasing function because $f^{\prime}(s)<0$. It suffices to consider (9) to see that $g^{\prime}$ is decreasing. Now we use (9) together with (H2) in order to obtain a lower bound on $g^{\prime}$ :

$$
g^{\prime}(s) \geq\left[\frac{\alpha}{1-\alpha} \gamma^{\frac{\alpha}{1-\alpha}}+\beta^{-1}\right]^{-1}(1+s)^{-\alpha} .
$$


Now we define the function

$$
\Psi(r, s)=(g(s)-g(r))(s-r)^{-1}(1+r+s)^{\alpha} .
$$

If we prove there exist two positive constants $\lambda_{1}$ and $\lambda_{2}$ satisfying

$$
\lambda_{1} \leq \Psi(r, s) \leq \lambda_{2} \quad \text { for all } r, s \in(0, \infty)
$$

then property (H3) will be satisfied. Moreover, since $\Psi(r, s)=\Psi(s, r)$, we can consider in the following only the case $s>r$.

In order to obtain the lower bound $\lambda_{1}$, it suffices to write:

$$
g(s)-g(r)=g^{\prime}(\xi)(s-r)
$$

where $\xi$ belongs to the interval with extremities $r$ and $s$. From (10) and because $g^{\prime}$ is decreasing, we obtain

$$
\begin{aligned}
g(s)-g(r) & \geq\left[\frac{\alpha}{1-\alpha} \gamma^{\frac{\alpha}{1-\alpha}}+\beta^{-1}\right]^{-1}(1+s)^{-\alpha}(s-r) \\
& \geq\left[\frac{\alpha}{1-\alpha} \gamma^{\frac{\alpha}{1-\alpha}}+\beta^{-1}\right]^{-1}(1+r+s)^{-\alpha}(s-r)
\end{aligned}
$$

which implies the lower bound $\lambda_{1}=\left[\frac{\alpha}{1-\alpha} \gamma^{\frac{\alpha}{1-\alpha}}+\beta^{-1}\right]^{-1}$.

In order to prove the upper bound, we use properties (H1, H2) and we verify the following relationships:

$$
\begin{aligned}
\Psi(r, s) & =(s f(s)-r f(r))(s-r)^{-1}(1+r+s)^{\alpha} \\
& =\left[f(s)+r(f(s)-f(r))(s-r)^{-1}\right](1+r+s)^{\alpha} \\
& \leq f(s)(1+r+s)^{\alpha} \leq f(s)(1+2 s)^{\alpha} \leq 2^{\alpha} \gamma .
\end{aligned}
$$

\section{Approximations ANd a PRIORI ERror ESTIMATES}

In this section we set $X=W_{0}^{1,2-\alpha}(\Omega)$ and the natural Sobolev norm in $X$ will be denoted by $\|\cdot\|_{W^{1,2-\alpha}(\Omega)}$ which is equivalent to $\|\cdot\|=\left(\int_{\Omega}|\nabla \cdot|^{2-\alpha} \mathrm{d} x\right)^{1 /(2-\alpha)}$. The weak formulation of problem $(1,2)$ corresponding to the Euler equation of the minimization of $J$ on $X$ is the following one: we are looking for $u \in X$ satisfying

$$
\int_{\Omega} f(|\nabla u|) \nabla u . \nabla v \mathrm{~d} x=\int_{\Omega} p v \mathrm{~d} x \quad \text { for all } v \in X .
$$

In order to establish a Galerkin approximation of (13) we choose a family of finite dimensional subspaces $X_{h}$ of $X$ satisfying

$$
\lim _{h \rightarrow 0} \min _{v_{h} \in X_{h}}\left\|v-v_{h}\right\|=0, \quad \forall v \in X
$$

and we are looking for $u_{h} \in X_{h}$ such that:

$$
\int_{\Omega} f\left(\left|\nabla u_{h}\right|\right) \nabla u_{h} . \nabla v_{h} \mathrm{~d} x=\int_{\Omega} p v_{h} \mathrm{~d} x \quad \text { for all } v_{h} \in X_{h} .
$$


By substracting (15) to (13), we obtain:

$$
\int_{\Omega}\left(f(|\nabla u|) \nabla u-f\left(\left|\nabla u_{h}\right|\right) \nabla u_{h}\right) . \nabla v_{h} \mathrm{~d} x=0 \quad \text { for all } v_{h} \in X_{h} .
$$

As a consequence we will have the following relationship:

$$
\begin{aligned}
& \int_{\Omega}\left(f(|\nabla u|) \nabla u-f\left(\left|\nabla u_{h}\right|\right) \nabla u_{h}\right) \cdot\left(\nabla u-\nabla u_{h}\right) \mathrm{d} x= \\
& \qquad \int_{\Omega}\left(f(|\nabla u|) \nabla u-f\left(\left|\nabla u_{h}\right|\right) \nabla u_{h}\right) \cdot\left(\nabla u-\nabla v_{h}\right) \mathrm{d} x \quad \text { for all } v_{h} \in X_{h}
\end{aligned}
$$

Now let us introduce a quasi-norm $|\cdot|_{\alpha}$ as in [2] by

$$
|v|_{\alpha}=\left\|(1+|\nabla u|+|\nabla v|)^{-\alpha / 2}|\nabla v|\right\|_{L^{2}(\Omega)},
$$

where $\|\cdot\|_{L^{2}(\Omega)}$ is the quadratic norm and $u$ is the solution of (13). $|v|_{\alpha}$ is not a norm because the homogeneity property is missing. However the other properties of the norm are true. From this definition together with properties (17) and (H3) we obtain:

Theorem 1. We assume that the function $f$ satisfies (3) and let $u$ and $u_{h}$ be the solutions of (13) and (15) respectively. Then there exists a constant $C$ such that the following error estimate holds:

$$
\left|u-u_{h}\right|_{\alpha} \leq C\left|u-v_{h}\right|_{\alpha}, \quad \forall v_{h} \in X_{h}
$$

Proof. We can find the proof of this result in [2], Theorem 1 and we don't completely repeat it here. Let us just mention a sketch of the proof that it is essentially a consequence of property (H3).

Since (H3) is satisfied (see Lem. 2), then the following relationships are true (see for instance [2]):

$$
\langle f(|\xi|) \xi-f(|\eta|) \eta, \xi-\eta\rangle \geq \chi(1+|\xi|+|\eta|)^{-\alpha} \cdot|\xi-\eta|^{2}
$$

and

$$
|f(|\xi|) \xi-f(|\eta|) \eta| \leq C(1+|\xi|+|\eta|)^{-\alpha}|\xi-\eta|
$$

for all $\xi, \eta \in R^{2}$, where $\langle.,$.$\rangle is the scalar product and |$.$| is the Euclidian norm in R^{2}$. Since, for $\xi, \eta \in R^{2}$ we have the obvious inequalities $\frac{1}{2}(|\xi|+|\eta|) \leq|\xi|+|\xi-\eta| \leq 2(|\xi|+|\eta|)$, we obtain by using (18) with $v=u-u_{h}$ and successively $(20,17,21)$ :

$$
\begin{aligned}
\left|u-u_{h}\right|_{\alpha}^{2} & =\int_{\Omega}\left(1+|\nabla u|+\left|\nabla u-\nabla u_{h}\right|\right)^{-\alpha}\left|\nabla u-\nabla u_{h}\right|^{2} \mathrm{~d} x \\
& \leq C \int_{\Omega}\left(f(|\nabla u|) \nabla u-f\left(\left|\nabla u_{h}\right|\right) \nabla u_{h}\right) \cdot\left(\nabla u-\nabla u_{h}\right) \mathrm{d} x \\
& \leq C \int_{\Omega}\left(f(|\nabla u|) \nabla u-f\left(\left|\nabla u_{h}\right|\right) \nabla u_{h}\right) \cdot\left(\nabla u-\nabla v_{h}\right) \mathrm{d} x \\
& \leq C \int_{\Omega}\left(1+|\nabla u|+\left|\nabla u_{h}\right|\right)^{-\alpha}\left|\nabla u-\nabla u_{h}\right|\left|\nabla u-\nabla v_{h}\right| \mathrm{d} x \\
& \leq C \int_{\Omega}\left(1+|\nabla u|+\left|\nabla\left(u-u_{h}\right)\right|\right)^{\alpha}\left|\nabla\left(u-u_{h}\right)\right| \cdot\left|\nabla\left(u-v_{h}\right)\right| \mathrm{d} x .
\end{aligned}
$$


By using inequalities $(1+a+r)^{-\alpha} r s \leq \varepsilon(1+a+r)^{-\alpha} r^{2}+\frac{1}{\varepsilon}(1+a+s)^{-\alpha} s^{2}$ for all $a, r, s \geq 0$ and $\left.\varepsilon \in\right] 0,1[$, by setting $a=|\nabla u|, r=\left|\nabla\left(u-u_{h}\right)\right|, s=\left|\nabla\left(u-v_{h}\right)\right|$ and by choosing $\varepsilon$ small enough, we obtain

$$
\left|u-u_{h}\right|_{\alpha}^{2} \leq C\left|u-v_{h}\right|_{\alpha}^{2}
$$

where $C$ denotes a generic constant independent of the parameter $h$.

In order to obtain error estimates in classical Sobolev spaces, we can use Holder inequalities applying to the quasi-norm $|\cdot|_{\alpha}$ as in [2]. If $\sigma \in[2-\alpha, 2]$ we have:

$$
M\|\nabla v\|_{L^{2-\alpha}(\Omega)}^{2} \leq|v|_{\alpha}^{2} \leq C\|\nabla v\|_{L^{\sigma}(\Omega)}^{\sigma},
$$

for all $v \in W_{0}^{1, \sigma}(\Omega)$ satisfying $\|\nabla v\|_{L^{2-\alpha}(\Omega)} \leq D$, where $D, C, M$ are positive constants $(M$ is depending on $D)$. From (23), together with the fact that $\lim _{h \rightarrow 0}\left\|u-u_{h}\right\|_{W_{0}^{1,2-\alpha}(\Omega)}=0$ and Theorem 1, we can easily prove the following result:

Theorem 2. We assume that the function $f$ satisfies (3) and let $u$ and $u_{h}$ be the solutions of (13) and (15) respectively. If $\sigma \in[2-\alpha, 2]$, if $u \in W_{0}^{1, \sigma}(\Omega)$ and if $X_{h} \subset W_{0}^{1, \sigma}(\Omega)$ then there exists a constant $C$ such that the following error estimate holds:

$$
\left\|\nabla\left(u-u_{h}\right)\right\|_{L^{2-\alpha}(\Omega)} \leq C\left\|\nabla\left(u-v_{h}\right)\right\|_{L^{\sigma}(\Omega)}^{\sigma / 2}, \quad \forall v_{h} \in X_{h} .
$$

Remark 1. Assume that $X_{h}$ is a finite element subspace of $H_{0}^{1}(\Omega)$ made of piecewise polynomial functions of degree 1 on each triangle of a regular triangulation $\Upsilon_{h}$ of $\bar{\Omega}$. It is well known (see Ciarlet [4]) that we have the following error estimates when $\sigma \in[2-\alpha, 2]$ :

$$
\min _{v_{h} \in X_{h}}\left\|\nabla u-\nabla v_{h}\right\|_{L^{\sigma}(\Omega)} \leq C h \quad \text { if } u \in W^{2, \sigma}(\Omega)
$$

where $h$ is the maximum of diameters of triangles contained in $\Upsilon_{h}$.

Following Theorem 2, we will obtain in this case:

$$
\left\|u-u_{h}\right\|_{W^{1,2-\alpha}(\Omega)} \leq C h^{1-\alpha / 2} \quad \text { if } u \in W^{2,2-\alpha}(\Omega),
$$

and

$$
\left\|u-u_{h}\right\|_{W^{1,2-\alpha}(\Omega)} \leq C h \quad \text { if } u \in H^{2}(\Omega) .
$$

Let us remark that with the same techniques used in Glowinski-Marrocco [6] for the $p$-Laplacian problem with $p=2-\alpha$, we can prove that function $f$ given by (3) satisfies the two following inequalities:

$$
\langle f(|\xi|) \xi-f(|\eta|) \eta, \xi-\eta\rangle \geq \chi \min \left(|\xi|^{-\alpha},|\eta|^{-\alpha}, 1\right) \cdot|\xi-\eta|^{2}
$$

and

$$
|f(|\xi|) \xi-f(|\eta|) \eta| \leq C|\xi-\eta|^{1-\alpha},
$$

for all $\xi, \eta \in R^{2}$, where $\langle. .,$.$\rangle is the scalar product and |$.$| is the Euclidian norm in R^{2}$.

From (17), (27) and (28) we easily prove by using a Holder inequality that the following estimate holds:

$$
\left\|\nabla\left(u-u_{h}\right)\right\|_{L^{2-\alpha}(\Omega)} \leq C\left\|\nabla\left(u-v_{h}\right)\right\|_{L^{2-\alpha}(\Omega)}^{1 /(1+\alpha)}, \quad \forall v_{h} \in X_{h} .
$$


As a consequence, we obtain with this argument an error estimate given by $\left\|\nabla\left(u-u_{h}\right)\right\|_{L^{2-\alpha}(\Omega)} \leq h^{1 /(1+\alpha)}$ if $u \in W^{2,2-\alpha}(\Omega)$, which is lesser order than the one in $(25)$ since $1 /(1+\alpha) \leq 1-\alpha / 2$.

\section{Approximations AND a POSTERIORI ERROR ESTIMATES}

In this paragraph, we assume that $X_{h}$ is a finite element subspace of $H_{0}^{1}(\Omega)$ made of piecewise polynomial functions of degree 1 on each triangle $K$ of a regular triangulation $\Upsilon_{h}$ of $\bar{\Omega}$. The parameter $h$ represents the maximum of diameters of triangles contained in $\Upsilon_{h}$. In order to establish a posteriori error estimates, we follow the paper by Baranger and El Amri [1], in which we can find residuals error estimates for the $p$-Laplacian model.

Let us define the residual quantity $R \in W^{-1, \frac{2-\alpha}{1-\alpha}}(\Omega)$ by

$$
\langle R, v\rangle_{X^{\prime} X}=\int_{\Omega} f\left(\left|\nabla u_{h}\right|\right) \nabla u_{h} . \nabla v \mathrm{~d} x-\int_{\Omega} p v \mathrm{~d} x \quad \forall v \in X
$$

where here, $\langle., .\rangle_{X^{\prime} X}$ is the duality pairing between $X^{\prime}=W^{-1, \frac{2-\alpha}{1-\alpha}}(\Omega)$ and $X=W_{0}^{1,2-\alpha}(\Omega)$.

As in Baranger-El Amri [1], we obtain by integrating by parts:

$$
\begin{aligned}
\langle R, v\rangle_{X^{\prime} X} & =\sum_{K \in \Upsilon_{h}}\left(\int_{K} f\left(\left|\nabla u_{h}\right|\right) \nabla u_{h} . \nabla v \mathrm{~d} x-\int_{K} p v \mathrm{~d} x\right) \\
& =\sum_{K \in \Upsilon_{h}}\left(-\int_{K} \operatorname{div}\left(f\left(\left|\nabla u_{h}\right|\right) \nabla u_{h}\right) v \mathrm{~d} x-\int_{K} p v \mathrm{~d} x+\int_{\partial K} f\left(\left|\nabla u_{h}\right|\right) \frac{\partial u_{h}}{\partial n} v \mathrm{~d} s\right) .
\end{aligned}
$$

Since $\left\langle R, v_{h}\right\rangle_{X^{\prime} X}=0$ for all $v_{h} \in X_{h}$, we write:

$$
\begin{aligned}
\langle R, v\rangle_{X^{\prime} X}= & \sum_{K \in \Upsilon_{h}}\left(-\int_{K} \operatorname{div}\left(f\left(\left|\nabla u_{h}\right|\right) \nabla u_{h}\right)\left(v-\pi_{h} v\right) \mathrm{d} x\right. \\
& \left.-\int_{K} p\left(v-\pi_{h} v\right) \mathrm{d} x+\int_{\partial K} f\left(\left|\nabla u_{h}\right|\right) \frac{\partial u_{h}}{\partial n}\left(v-\pi_{h} v\right) \mathrm{d} s\right),
\end{aligned}
$$

where $\pi_{h}$ is the Clement's interpolation operator.

By using the approximation properties of $\pi_{h}$ we prove by following the same arguments given by BarangerEl Amri [1] that:

$$
\|R\|_{X^{\prime}}=\sup _{\|v\|=1}\langle R, v\rangle_{X^{\prime} X} \leq C\left(\sum_{K \in \Upsilon_{h}} \eta^{m}(K)\right)^{1 / m} \quad \text { with } m=\frac{2-\alpha}{1-\alpha}
$$

where the estimator $\eta(K)$ is given by

$$
\eta(K)=\left\{h_{K}^{m}\left\|\operatorname{div}\left(f\left(\left|\nabla u_{h}\right|\right) \nabla u_{h}\right)+p\right\|_{L^{m}(K)}^{m}+\sum_{t \in \partial K} h_{t}\left\|\left[f\left(\left|\nabla u_{h}\right|\right) \frac{\partial u_{h}}{\partial n}\right]_{t}\right\|_{L^{m}(\partial K)}^{m}\right\}^{1 / m} .
$$

In the above expression, $h_{K}$ is the diameter of $K, h_{t}$ is the length of the side $t \in \partial K$ and $[\cdot]_{t}$ denotes the jump through the side $t$ of the triangle $K$. If the measure of $\partial K \cap \partial \Omega$ is not vanishing, the jump $[\cdot]_{t}$ is only defined by the internal value in $\Omega$. At this point, let us remark that the power of $h_{K}$ in the estimator $\eta(K)$ of [1] contains a misprint. 
Clearly speaking in our estimator $\eta(K)$, since $\nabla u_{h}$ is constant on each triangle $K$, the term $\operatorname{div}\left(f\left(\left|\nabla u_{h}\right|\right) \nabla u_{h}\right)$ is vanishing and we obtain:

$$
\eta(K)=\left\{h_{K}^{m}\|p\|_{L^{m}(K)}^{m}+\sum_{t \in \partial K} h_{t}\left\|\left[f\left(\left|\nabla u_{h}\right|\right) \frac{\partial u_{h}}{\partial n}\right]_{t}\right\|_{L^{m}(\partial K)}^{m}\right\}^{1 / m} .
$$

Now we are able to prove the following a posteriori error estimate:

Theorem 3. We assume that the function $f$ satisfies (3) and let $u$ and $u_{h}$ be the solutions of (13) and (15) respectively. Then there exists a constant $C$ such that:

$$
\left\|\nabla\left(u-u_{h}\right)\right\|_{L^{2-\alpha}(\Omega)} \leq C\left(\sum_{K \in \Upsilon_{h}} \eta^{m}(K)\right)^{1 / m}, \quad \text { with } m=\frac{2-\alpha}{1-\alpha} .
$$

Proof. We have to link the error $\left\|u-u_{h}\right\|$ to $\|R\|_{X^{\prime}}$. From the definition of $R$ and since $u$ is the solution of problem (1, 2), we have:

$$
\langle R, v\rangle_{X^{\prime} X}=\int_{\Omega}\left(f\left(\left|\nabla u_{h}\right|\right) \nabla u_{h}-f(|\nabla u|) \nabla u\right) \cdot \nabla v \mathrm{~d} x
$$

and consequently

$$
\|R\|_{X^{\prime}}=\left\|f\left(\left|\nabla u_{h}\right|\right) \nabla u_{h}-f(|\nabla u|) \nabla u\right\|_{L^{m}(\Omega)} .
$$

From (22) and (23) we obtain:

$$
\begin{aligned}
\left\|\nabla\left(u-u_{h}\right)\right\|_{L^{2-\alpha}(\Omega)}^{2} & \leq C \int_{\Omega}\left(f(|\nabla u|) \nabla u-f\left(\left|\nabla u_{h}\right|\right) \nabla u_{h}\right) \cdot\left(\nabla u-\nabla u_{h}\right) \mathrm{d} x \\
& \leq C\left\|f\left(\left|\nabla u_{h}\right|\right) \nabla u_{h}-f(|\nabla u|) \nabla u\right\|_{L^{m}(\Omega)} \cdot\left\|\nabla\left(u-u_{h}\right)\right\|_{L^{2-\alpha}(\Omega)} .
\end{aligned}
$$

It follows that $\left\|\nabla\left(u-u_{h}\right)\right\|_{L^{2-\alpha}(\Omega)} \leq C\|R\|_{X^{\prime}}$.

\section{NumericAl RESULTS}

In this paragraph, we give some numerical results in order to illustrate the a priori and a posteriori error estimates results obtained in Sections 3 and 4.

\section{A priori error estimates}

We start by showing that the error estimate (26) we obtained in Section 3 is optimal if the solution is regular (say $u \in H^{2}(\Omega)$ ). However we will see that the error estimate (25) is not optimal in the case of non-regular solutions. To do this, we have chosen $\alpha=1 / 2$ which leads to the explicit function:

$$
f(s)=2\left[T_{0}+\sqrt{T_{0}^{2}+4 s}\right]^{-1} .
$$

Starting from a given function $u\left(x_{1}, x_{2}\right)$, we can compute

$$
p=-\operatorname{div}(f(|\nabla u|) \nabla u) .
$$




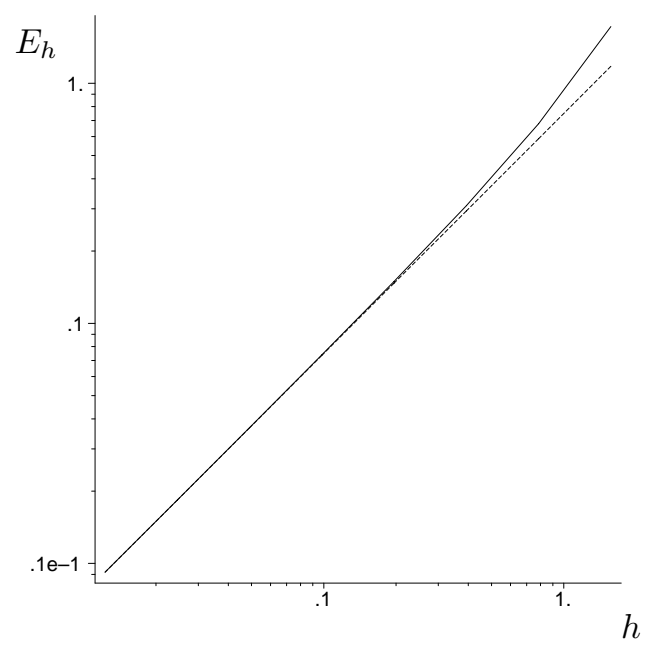

Figure 1. A priori error estimate $E_{h}$ in function of $h$ when $u\left(x_{1}, x_{2}\right)=\sin \left(x_{1}\right) \sin \left(x_{2}\right)$. (The dotted line is a straightline with slope 1.)

After solving approximate problem (15) with that function $p$, we can plot in a log-log diagram the a priori error $E_{h}$ defined by

$$
E_{h}=\left\|\nabla u-\nabla u_{h}\right\|_{L^{3 / 2}(\Omega)} .
$$

The slope of the graph of $E_{h}$ as a function of $h$ in log-log scale gives the rate of convergence.

As an example we chose the domain $\Omega=(0, \pi) \times(0, \pi)$ and the function $u\left(x_{1}, x_{2}\right)=\left(\sin \left(x_{1}\right) \sin \left(x_{2}\right)\right)$ which is in the space $H^{2}(\Omega)$. The square $\Omega$ is divided into $N \times N$ equal squares and each square is splitted into two triangles by its diagonal with direction $(1,1)$. In Figure 1, we represent the a priori error in a log-log scale when the approximation space $X_{h}$ is the finite element space of degree 1 on this triangulation. We observe a rate of convergence of order $h$ like predicted by $(26)$.

If we choose the function $u\left(x_{1}, x_{2}\right)=\left(\sin \left(x_{1}\right) \sin \left(x_{2}\right)\right)^{1.34}$ which is in the space $W_{0}^{2, \frac{3}{2}}(\Omega)$ but not in $W_{0}^{2,1.6}(\Omega)$, we observe in Figure 2 a rate of convergence of order $h^{0.95}$ which shows that the error estimate $(25)$ is not optimal. In fact error estimate (25) gives in this case a rate of convergence of order $h^{\frac{3}{4}}=h^{0.75}$ which is less accurate than $h^{0.95}$.

\section{A posteriori error estimates}

As above, we consider the case $\alpha=1 / 2$ which allows us to obtain an explicit expression for $f(s)$. We $a$ priori fix the solution $u$ and we compute the right-hand side $p$ which corresponds to $u$. On the boundary $\partial \Omega$ of the unit square $\Omega=(0,1) \times(0,1)$ we prescribe a Dirichlet condition (not necessary homogeneous) for $u$ which doesn't change our theoretical results.

In Table 1 we can see the true error $E_{h}=\left\|\nabla u-\nabla u_{h}\right\|_{L^{3 / 2}(\Omega)}$, the estimated error $E_{s}=\left(\sum_{K \in \Upsilon_{h}} \eta^{3}(K)\right)^{1 / 3}$ and the effectivity index $E_{s} / E_{h}$ in the case where $u=x_{1}^{2}+x_{2}^{2}$. As above, the square $\Omega$ has been divided into $N \times N$ equal squares and each square has been splitted into two triangles by its diagonal with direction $(1,1)$. We set $h=1 / N$.

As we observe in Table 1, the effectivity index is very close to 2 and doesn't depend on $h$. Except the factor 2 , our estimated error $E_{s}$ seems to be a good estimation of the error $E_{h}$. 


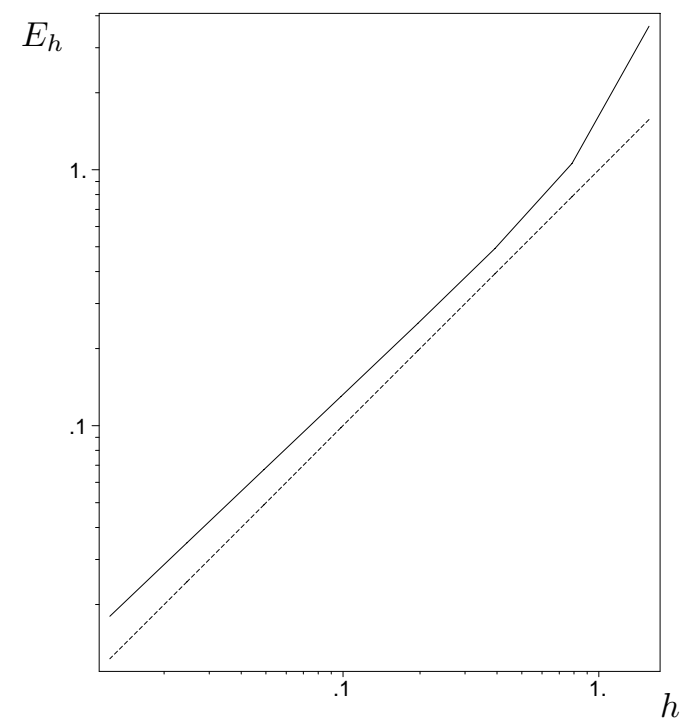

Figure 2. A priori error estimate $E_{h}$ in function of $h$ when $u\left(x_{1}, x_{2}\right)=\left(\sin \left(x_{1}\right) \sin \left(x_{2}\right)\right)^{1.34}$. (The dotted line is a straightline with slope 1.)

TABLE 1. True error $E_{h}=\left\|\nabla\left(u-u_{h}\right)\right\|_{L^{3 / 2}(\Omega)}$, estimated error $E_{s}=\left(\sum_{K \in \Upsilon_{h}} \eta^{m}(K)\right)^{1 / m}$ and effectivity index $E_{s} / E_{h}$.

\begin{tabular}{|r|r|r|r|r|}
\hline$h$ & vertices & $E_{h}$ & $E_{s}$ & $E_{s} / E_{h}$ \\
\hline $1 / 5$ & 36 & 0.07634 & 0.1414 & 1.853 \\
$1 / 10$ & 121 & 0.03816 & 0.07391 & 1.937 \\
$1 / 20$ & 441 & 0.01908 & 0.03775 & 1.979 \\
$1 / 40$ & 1681 & 0.009540 & 0.01907 & 1.999 \\
$1 / 80$ & 6561 & 0.004771 & 0.009586 & 2.009 \\
$1 / 160$ & 25921 & 0.002389 & 0.004805 & 2.011 \\
$1 / 320$ & 103041 & 0.001220 & 0.002405 & 1.972 \\
\hline
\end{tabular}

In Table 2 we have computed the estimated error $\tilde{E}_{s}$ given by Liu and Yan [8] for the $p$-Laplacian. In this example where $\alpha=1 / 2$, we have

$$
\tilde{E}_{s}=\left(\sum_{K \in \Upsilon_{h}} \tilde{\eta}^{2}(K)\right)^{1 / 2}
$$

where

$$
\begin{aligned}
\tilde{\eta}^{2}(K)= & \int_{K}\left(\left|\nabla u_{h}\right|^{1 / 2}+h_{K}|p|\right) h_{K}^{2}|p|^{2} \mathrm{~d} x \\
& +\sum_{t \in \partial K} \int_{K_{t}}\left(\left|\nabla u_{h}\right|^{1 / 2}+\left[\left|\nabla u_{h}\right|^{-1 / 2} \frac{\partial u_{h}}{\partial n}\right]\right)\left[\left|\nabla u_{h}\right|^{-1 / 2} \frac{\partial u_{h}}{\partial n}\right]^{2} \mathrm{~d} x .
\end{aligned}
$$


TABLE 2. True error $E_{h}=\left\|\nabla\left(u-u_{h}\right)\right\|_{L^{3 / 2}(\Omega)}$, estimated error $\tilde{E}_{s}=\left(\sum_{K \in \Upsilon_{h}} \tilde{\eta}^{2}(K)\right)^{1 / 2}$ and effectivity index $\tilde{E}_{s} / E_{h}$.

\begin{tabular}{|r|r|r|r|r|}
\hline$h$ & vertices & $E_{h}$ & $\tilde{E}_{s}$ & $\tilde{E}_{s} / E_{h}$ \\
\hline $1 / 5$ & 36 & 0.07634 & 0.2152 & 2.819 \\
$1 / 10$ & 121 & 0.03816 & 0.1330 & 3.485 \\
$1 / 20$ & 441 & 0.01908 & 0.07271 & 3.811 \\
$1 / 40$ & 1681 & 0.009540 & 0.03792 & 3.975 \\
$1 / 80$ & 6561 & 0.004771 & 0.01936 & 4.058 \\
$1 / 160$ & 25921 & 0.002389 & 0.009778 & 4.093 \\
$1 / 320$ & 103041 & 0.001220 & 0.004913 & 4.028 \\
\hline
\end{tabular}

Here, we denote by $K_{t}$ the triangle satisfying

$$
\left|\nabla u_{h}\right|_{K_{t}}=\min _{i=1,2}\left(\left|\nabla u_{h}\right|_{K_{t}^{i}}\right)
$$

where $K_{t}^{1}, K_{t}^{2}$ are the two elements sharing the common side $t$.

If we compare Table 1 and Table 2 we can conclude that $E_{s}$ and $\tilde{E}_{h}$ are two estimators with some accuracy order (in $h$ ) but the effectivity index is better for $E_{s}$ (close to 2 ) than for $\tilde{E}_{s}$ (close to 4 ).

In the following, we present some numerical results related to an adaptive finite element method based on the a posteriori error estimate of Theorem 3. Our goal is now to build a mesh such that the estimated relative error is close to a preset tolerance Tol, namely

$$
0.75 \mathrm{Tol} \leq E_{s} /\left\|\nabla u_{h}\right\|_{L^{2-\alpha}(\Omega)} \leq 1.25 \mathrm{Tol}
$$

A sufficient condition to build such a mesh is to check that, for all triangle $K \in \Upsilon_{h}$ we have

$$
\nu_{1} \frac{\left\|\nabla u_{h}\right\|_{L^{2-\alpha}(\Omega)}}{\left(N_{T}\right)^{1 / m}} \leq \eta(K) \leq \nu_{2} \frac{\left\|\nabla u_{h}\right\|_{L^{2-\alpha}(\Omega)}}{\left(N_{T}\right)^{1 / m}}
$$

where $\nu_{1}=0.75 \mathrm{Tol}, \nu_{2}=1.25 \mathrm{Tol}$ and $N_{\mathrm{T}}$ is the number of triangles in the mesh $\Upsilon_{h}$. The adaptive algorithm we have used is an iterative method which adds or suppresses some vertices in the triangulation in order to generate a new Delaunay-Voronoi triangulation satisfying (30) for the best.

To illustrate our purpose, we still choose $\alpha=1 / 2$ and for solution of (1), we choose a function $u$, the graph of which is very sharp in a neighborhood of the circle centered at the middle of the square $\Omega$ and with radius 0.2 . More precisely we choose $u=\exp \left((r-a)^{2} /\left((r-a)^{2}-\varepsilon^{2}\right)\right)$ if $a<r<a+\varepsilon$, with $r^{2}=x_{1}^{2}+x_{2}^{2}, \varepsilon=0.02$ and $a=0.2$. If $r<a$ we set $u=1$ and if $r>a+\varepsilon$ we set $u=0$. Figures 3 to 6 show the initial mesh and the meshes obtained after 1,5 and 10 iterations respectively. We conclude that the adaptive finite element method combined with the a posteriori error estimate given in Theorem 3 is efficient.

Acknowledgements. The authors would like to thank Adrian Reist for providing the numerical results given in Section 5 . The second author also thanks the Department of Computational and Applied Mathematics of Rice University and the Department of Mathematics of the University of Houston for their hospitality during his sabbatical stay. 


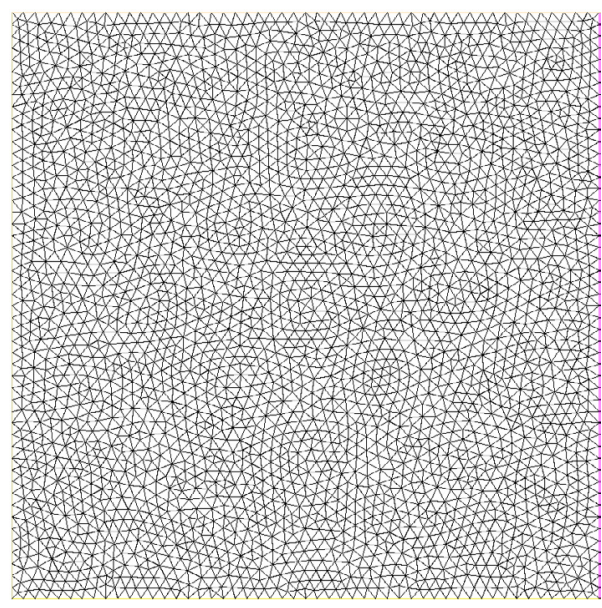

FiguRE 3. Initial mesh.

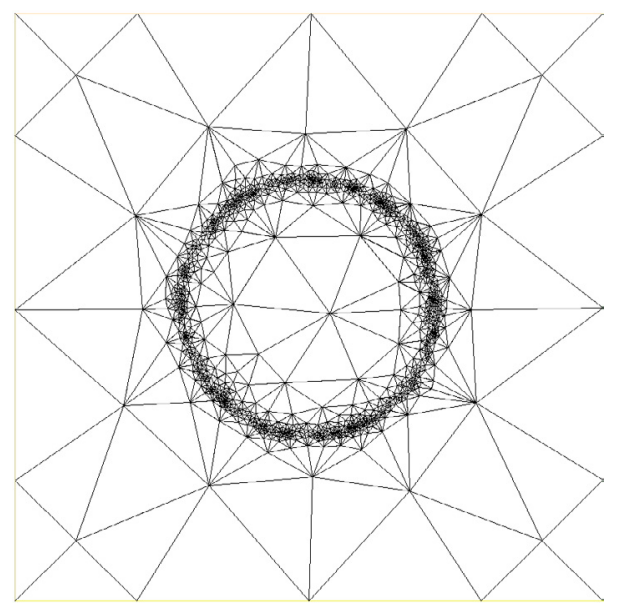

Figure 5. Mesh after five iterations.

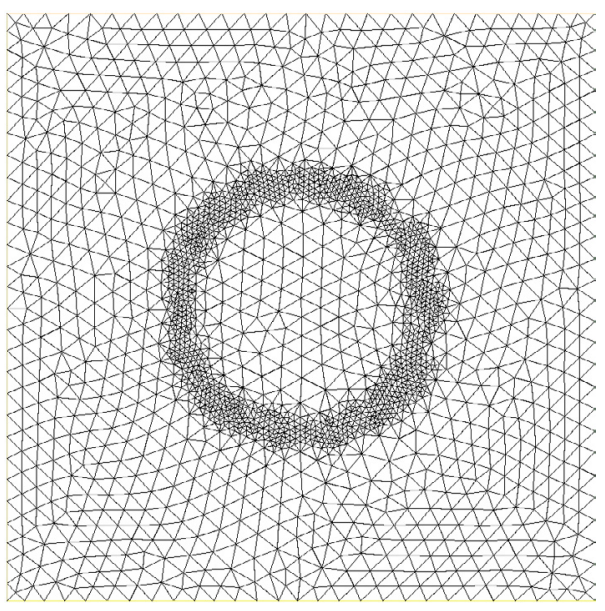

FIGURE 4. Mesh after one iteration.

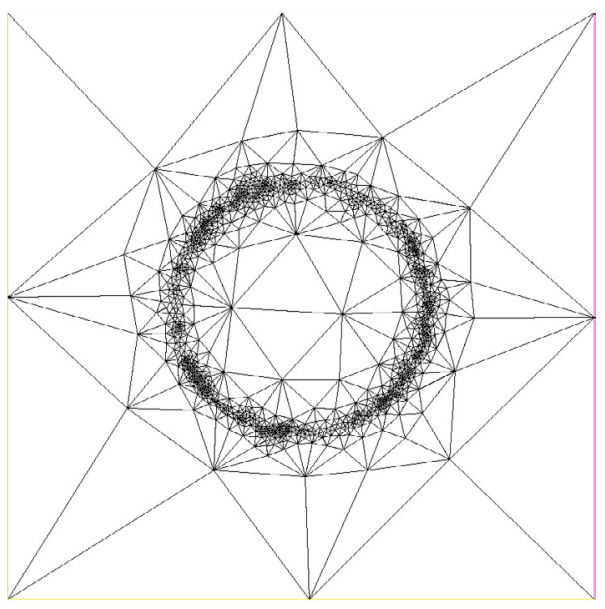

FiguRE 6. Mesh after ten iterations.

\section{REFERENCES}

[1] J. Baranger and H. El Amri. Estimateurs a posteriori d'erreurs pour le calcul adaptatif d'écoulements quasi-newtoniens. RAIRO Modél. Math. Anal. Numér. 25 (1991) 31-48.

[2] J.W. Barrett and W. Liu, Finite element approximation of degenerate quasi-linear elliptic and parabolic problems. Pitman Res. Notes Math. Ser. 303 (1994) 1-16. In Numerical Analysis 1993.

[3] H. Blatter, Velocity and stress fields in grounded glacier: a simple algorithm for including deviator stress gradients. J. Glaciol. 41 (1995) 333-344.

[4] P.G. Ciarlet, The finite element method for elliptic problems. North-Holland, Stud. Math. Appl. 4 (1978).

[5] J. Colinge and J. Rappaz, A strongly non linear problem arising in glaciology. ESAIM: M2AN 33 (1999) 395-406.

[6] R. Glowinski and A. Marrocco, Sur l'approximation par éléments finis d'ordre un, et la résolution par pénalisation-dualité, d'une classe de problèmes de Dirichlet non linéaires. Anal. Numér. 2 (1975) 41-76.

[7] P. Hild, I.R. Ionescu, T. Lachand-Robert and I. Rosca, The blocking of an inhomogeneous Bingham fluid. Applications to landslides. ESAIM: M2AN 36 (2002) 1013-1026.

[8] W. Liu and N. Yan. Quasi-norm local error estimators for p-Laplacian. SIAM J. Numer. Anal. 39 (2001) 100-127.

[9] A. Reist, Résolution numérique d'un problème à frontière libre issu de la glaciologie. Diploma thesis, Department of Mathematics, EPFL, Lausanne, Switzerland (2001). 